\title{
Biomaterials meet microfluidics: building the next generation of artificial niches
}

\author{
Stefan Kobel and Matthias P. Lutolf*
}

Laboratory of Stem Cell Bioengineering (LSCB) and Institute of Bioengineering (IBI), Ecole Polytechnique Fédérale de Lausanne (EPFL), Lausanne, Switzerland ("E-mail: matthias.lutolf@epfl.ch)

Biomaterials are increasingly being developed as in vitro microenvironments mimicking in vivo stem cell niches. However, current macro-scale methodologies to produce these niche models fail to recapitulate the spatial and temporal characteristics of the complex native stem cell regulatory systems. Microfluidic technology offers unprecedented control over the spatial and temporal display of biological signals and therefore promises new avenues for stem cell niche engineering. Here we discuss how the two approaches can be combined to generate more physiological models of stem cell niches that could facilitate the identification of new mechanisms of stem cell regulation, profoundly impacting drug discovery and ultimately therapeutic applications of stem cells.

\section{Introduction}

Stem cell biologists begin to recognize the relevance of advanced biomaterials as tools for the study of stem cell self-renewal and differentiation. The interest in biomaterials has risen largely due to the increased awareness of the shortcomings of existing cell culture techniques to control stem cell behavior in vitro. Isolated from a tissue and exposed to a rigid and hydrophobic plastic cell culture substrate, many adult stem cell types rapidly lose their multipotent characteristics. In vivo, the stem cells reside in highly hydrated and soft niches (Fig. 1A) that anchor the stem cell to its anatomical site in a tissue and provide instructive cues that regulate fate (Fig. 1B) [1,2]. Niches are typically composed of crosslinked extracellular matrices (ECMs) rich in proteins and sugars, as well as support cells that secrete short-range soluble signaling cues and provide crucial cell-cell interactions (Fig. 1A).

The niche-dependency of stem cell fate therefore necessitates in vitro milieus that provide the key biochemical signals that need to be presented in a tissue-specific, biophysically relevant context [3,4••]. Indeed, new generations of biomaterials can be tailor-made to mimic the in vivo milieu of stem cells [5,6,7] (Fig. 1C). Recent advances allow for example the fabrication of hydrogels with precisely and to some extend independently tunable mechanical property, degradability and bioactivity (e.g. [8,9•]). However, despite these exciting advances, many mammalian stem cells still cannot be cultured in vitro for an extended period of time without losing their self-renewing capacity, such as has been shown for hematopoietic stem cells [10] or muscle stem cells [4••], and the differentiation of stem cells in a highly controlled fashion remains a formidable challenge, presumably because of our limited control over the spatial and temporal presentation of biological signals to stem cells in vitro. 
We believe that one way to overcome this hurdle is the application of microfluidic technology to the design and modification of biomaterials. Microfluidics, that is the manipulation of nano- and picoliters of liquid within channels of dedicated micro-devices, is characterized by laminar flow profiles and diffusive mixing. It is this unique flow regime that allows the positioning of biomolecules or cells with previously unmet precision and well-defined dynamics $[11,12,13]$. When cleverly combined with biomolecular materials, this approach thus promises the development of a panoply of miniaturized models of stem cell microenvironments or even tissues with various levels of complexity (Fig. 1D). What is more, new ways of screening artificial microenvironments in high-throughput can be envisioned (Fig. 1E).

Here we review recent efforts that have integrated biomaterials, particularly hydrogels, in microfluidics platforms (or vice versa) to study stem cells in a physiologically relevant context. Furthermore, we highlight emerging technologies that have not yet found their way to stem cell applications, but, if successfully implemented, could become powerful tools to further our understanding of stem cell biology.

\section{Micropatterning of biomolecules and hydrogels on surfaces}

In the native niche, stem cells may be exposed to graded or 'polarized' microenvironmental distributions of biomolecules such as growth factors or ECM proteins. In vitro, an asymmetric signal distribution can for example be achieved on a surface by hydrodynamic patterning, which relies on the controllable diffusive mixing in micro-channels of microfluidic devices.

One the one hand, when employing a chip where diffusion is limited, hydrodynamic patterning can be directly used to stimulate cells in a spatially controlled fashion with bioactive molecules at sub-cellular resolution by flowing two different liquid streams over a single cell (as successfully demonstrated in classical experiments [14]), embryonic stem cell (ESC) colonies [15], or even individual Drosophila embryos [16]. On the other hand, by manipulating the diffusive mixing of two inlet streams, microfluidics allows the well-controlled generation of continuous gradients of soluble proteins. These microfluidic gradient generators offer higher spatial control than classical methods such as the Boyden chamber or micropipets. Consequently, numerous systems to generate gradients have been developed over the last decade (as reviewed in [17]) and utilized, for example, to investigate the effect of various soluble growth factor gradients on neural stem cell behavior [18].

However, most studies employing gradient makers were conducted with cells cultured on non-physiological glass or plastic substrates. Clearly, integrating biomimetic hydrogels into such hydrodynamic patterning devices would increase their relevance. This may be achieved by simply coating a chip surface with a biomaterial of interest [15], or by employing microfluidics to synthesize micro-patterned hydrogels directly within the micro-channels. To this end, step-wise photopolymerization reactions were for example used to 
generate cell-adhesive poly(ethylene glycol) (PEG) substrates with defined stiffness regions to investigate directional cell migration ('durotaxis') [19•]. Notably, at the end of the fabrication, the chip was removed and the patterned substrate used for cell culture in a normal macro-scale culture environment.

Alternatively, preformed reactive hydrogel films may be patterned by tagged biomolecules using microfluidic technology [20,21•] (Fig. 2). A PEG hydrogel film, covalently modified with NeutrAvidin and/or ProteinA, was for example used to capture from solution, in just a few minutes, gradients of biotinylated or Fc-tagged proteins within a microfluidic gradient maker (Fig. 2A-C). After patterning, the microfluidic chip was removed and the patterned hydrogels used for cell culture. Importantly, hydrodynamic flow focusing allowed forming, in a software-controlled fashion, gel-immobilized protein patterns with virtually any shape (Fig. 2D) [21•], while the orthogonality of the two affinity binding schemes enabled independent immobilization of two overlapping protein gradients (Fig. 2E). Hydrodynamic patterning of hydrogels could be applied to other elegant microfluidic techniques [22,23•].

\section{Micropatterning of proteins and hydrogels in three dimensions}

Whereas the above two-dimensional (2D) approaches provide powerful reductionist models to study the role of the heterogeneous distribution of biomolecules in a natural stem cell niche, they omit the third dimension and therefore possibly an important determinant of stem cell fate. However, exciting advances have been made in generating three-dimensional (3D) artificial niches by combining microfluidics and biomaterials technology.

In one popular approach, hydrogel precursors and cells can be loaded between two channels within a microfluidic device where the hydrogel crosslinks in situ and encapsulates the cells. The channels can then be perfused to act as a source and sink of a biomolecule diffusing through the hydrogel to establish a stable gradient. In order to perfuse the system, it is obviously crucial not to obstruct the perfusion channels with the hydrogel which requires localized gelation for example via photolithography [24,25], micro-patterning via laminar flow $[26 \bullet, 27]$ or by physical confinement. The latter is usually achieved with narrowly spaced posts, which, when properly designed, restrict the flow of the liquid gel precursor by balancing surface tension and capillary force [28,29,30 •] (Fig. 3). This chip design, which has been commercialized (e.g. Ibidi $\mathrm{GmbH}$, Germany) [31], has been successfully applied to study cells exposed to gradients in 3D gels [32,33]. Examples include studies of neurite growth in a 3D collagen gel under a Netrin-gradient [32], or the de novo formation of vascular capillaries under a gradient of vascular endothelial cell growth factor or sphingosine-1-phosphate [33].

\section{Biomicrofluidics: fabricating microchannel networks within bioactive hydrogels}

Most microfluidic chips are fabricated from PDMS, because this material has favorable properties such as ease of processing and handling, permeability to gases, and elastic deformability that enables for example the integration of on-chip valves in multilayered chips. Nevertheless, as a substrate and device for applications in stem cell niche engineering, PDMS has some limitations. It 
rapidly adsorbs proteins, is rather difficult to biofunctionalize and does not allow the encapsulation of cells. For these reasons, researchers have sought to directly pattern micro-channels within hydrogels such as alginate, agarose or gelatin [13]. These 'biomicrofluidic' devices can be readily perfused [34,35] and at the same time can serve as scaffolds for cells $[36,37,38]$. In contrast to solid PDMS chips, nutrients and proteins can almost freely diffuse, just as within native ECMs. 3D biomolecule gradients can thus be established in a near-physiological context $[34,35,38]$.

Albeit not yet frequently applied to stem cell biology, such hydrogel chips could offer exciting avenues to modulate the cellular microenvironment. Nonetheless, their current fabrication by micromolding against a PDMS template makes the fabrication of complex 3D networks or multi-layered cellular constructs difficult. In addition, reliable micromolding necessitates relatively highly crosslinked hydrogels that may not be well suited for 3D stem cell culture and the induction of a morphogenetic cellular process. One approach that could circumvent these limitations may be the use of inkjet printing to fabricate biomicrofluidic networks. Inkjet printing theoretically allows the deposition of pico-liter droplets containing biomaterial precursors, cells and growth factors $[39,40]$. If suitable 'bio-inks' were available, the fabrication of macroscopic, cell-containing 3D constructs based on computer-assisted designs could be envisioned. Such physiological 3D cell culture models could not only be highly relevant for basic biological studies, but also for in vitro drug screening and ultimately regenerative medicine.

\section{Microfluidic platforms for high-throughput screening of 3D artificial stem cell niches}

An important impetus for the application of microfluidic technology in the context of stem cell niche engineering is its huge potential for miniaturization and therefore parallelization of experiments [41]. For example, microenvironments could be microfluidically produced in high-throughput as miniaturized cell culture systems, for screening applications or as microtissues (Fig. 1E). One approach to achieve this goal consists in the development of integrated $3 \mathrm{D}$ chips with on-chip microfluidic valves. Such a system has been developed containing an array of 16 individually addressable cell-culture chambers that could be loaded with hydrogels and stem cells [42].

Alternatively, microfluidic flow-through devices could be used to generate microbeads composed of hydrogels, cells and test compounds. They could be produced using stop-and-flow systems [43,44] or via microfluidic droplet generation. The latter technology relies on the injection of an aqueous solution into a stream of a carrier fluid, typically oil, inside of a microfluidic chip. Because the liquid streams are not miscible, they reproducibly emulsify at a very high frequency where the two streams meet [45]. Methodologies are emerging to produce microdroplets containing liquid gel precursors that can be crosslinked to hydrogel microbeads in the presence of cells and, if necessary, containing a tag encoding the composition of each microbead $[43,46 \bullet, 47]$. However, at the moment, hydrogel 
microbeads have mostly been fabricated from naturally derived polymers such as alginate that polymerize rapidly and are relatively dense (e.g. $[34,48 \cdot, 49,50])$; slowly polymerizing and soft hydrogel systems require extensive incubation channels (in flow-through system, incubation time translates into distance) [51]. This limits the use of microbead technology for some stem cell types that grow poorly in densely crosslinked hydrogel systems. Therefore, microfluidic set-ups and biomaterials will need to be further optimized to produce cell-permissive microbeads in high-throughput.

Nevertheless, over the last five years or so, microdroplet technology has progressed at impressive pace. Microdroplets can now be reliably stored, fused, separated and analyzed [45], and there are methods to modulate the composition of the microdroplets in high-throughput $[46 \bullet \cdot, 52 \cdot \bullet]$. To this end, a microfluidic chip was developed to screen a library of eight acetylenes and 16 acides under four different conditions for click crosslinking reactions $[46 \cdot \bullet, 52 \cdot \bullet]$. The 1024 combinations were prepared and mixed on the microfluidic chip in a fully automated fashion and then sequentially loaded into Teflon tubing for incubation. From there, each combination could be eluded, purified and analyzed by mass spectrometry. Intriguingly, the preparation of a single combination required only ca. 17 seconds. The application of similar strategies to screen for hydrogel-based stem cell microenvironments to discover functional artificial niches would be truly exciting.

A different approach was recently described to screen the effect of variable concentrations of the chematherapeutic agent mitomycine $C$ on nonadherent cancer cells [46••]. The authors first prepared a droplet-based library of different drug concentrations that was labeled with a fluorescent tag. This library was then loaded onto a second microfluidic chip where the droplets containing the cytotoxic compound were fused pair-wise with microdroplets containing the cells. After removing the microdroplets from the chip and incubating them for four days, the microdroplets were loaded onto a third chip and cell viability and the fluorescence tag were analyzed using spectroscopic methods.

The successful integration of hydrogel microbeads could extend such screening possibilities to adherent (stem) cell types. One could imagine either drug screens or combinatorial testing of different ECM compositions. That said, screening applications will surely not be the only application of such microbeads as they could for example be utilized as stem cell expansion carriers, building blocks for tissue constructs or implantable bio-sensors $[53,54,55]$.

\section{Towards functional tissue models using microfluidic chips and biomaterials}

One of the most captivating challenges in modern tissue engineering is the in vitro reconstruction of tissue or organ function. Microfluidics is well poised to facilitate such applications. Recently, an 'artificial lung' was generated on a chip composed of an immunologically functional bi-layer of endothelial and epithelial cells seeded onto the two sides of a porous PDMS membrane [56・•]. This device served as a realistic model to study the toxicity of nanoparticles 
by mimicking the respiration-induced deformation of the bi-layer and thus could trigger the strain-dependent uptake of nano-particles by the epithelium.

An application of this concept to 3D tissue models, such as the bone marrow would require the integration of advanced biomaterials. We believe that most of the approaches that were discussed above could be suitable for this purpose. For example, photo-polymerizable materials can easily be patterned into complex structures using photolithography [24,25,57], or microfluidics can be used to assemble micro-tissues in a precise configuration for 'tissue-on-a-chip' models [54]. Alternatively, microfluidically generated hydrogel microbeads might be directly used to assemble three-dimensional tissue-models off-chip [58].

An important challenge in the fabrication of functional artificial tissues is the adequate 3D patterning of multiple cell types into a tissue-like architecture. Microfluidics offers precise spatial control of the components of 3D co-culture systems [28,29,30•]. For example, researchers have engineered a hydrogel bi-layer of hepatocytes and endothelial cells. Interestingly, the system allowed the mimicry of interstitial flow which promoted the formation of tissue-like structures by the hepatocytes [59]. Similar work has recently yielded a recapitulation of the perivascular association of mesenchymal stem cells (MSCs) [30•]. 400- $\mu$ m wide stripes of hydrogels were loaded with mesenchymal stem cells (MSCs) with human umbilical vein endothelial cells (HUVECs) seeded to the channels next to the hydrogels. Over 14 days, these endothelial cells vascularized the hydrogels, while MSCs started to form close associations with the new capillaries. Although this represents only a first step towards the in vitro recapitulation of the perivascular niche of adult stem cells, such microfabricated co-culture models could be further advanced for example by applying biomolecules gradients, or by seeding primary functional niche cells, such as Nestin ${ }^{+}$MSCs [60], together with functional hematopoietic stem cells.

\section{Conclusions}

The fields of biomaterials engineering and microfluidics have advanced to impressive levels of sophistication. The interface of these two fields now promises truly exciting avenues for stem cell biotechnology and biology. Novel in vitro platforms to decipher the mechanism of stem cell regulation have already been generated. For example, the micropatterning of biomaterials using microfluidics has made possible the fabrication of physiological niche models in two and three dimensions. These models are not only able to mimic the asymmetric or graded distribution of key niche components, but also the precise 3D arrangement of different cell types in co-culture systems. The time is ripe to expand on these pioneering efforts to further integrate biomaterials technology into microfluidic chips to tackle pertinent questions in stem cell research. The results of these efforts might have impact beyond in vitro studies into regenerative therapies of the future.

\section{Acknowledgments}

We thank our collaborators in the Laboratory of Stem Cell Bioengineering, namely Steffen Cosson and Simone Allazetta, for inspiring discussions. We 
acknowledge the Swiss National Science Foundation grants CR32I3 125426 and CR23I2_125290 as well as a European Young Investigator (EURYI) Award (PE002-117115/1) to M.P.L

References and recommended reading 
Figure 1:

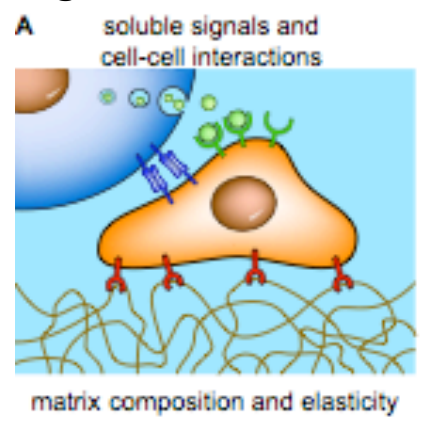

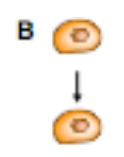

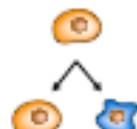

asymmetric

division
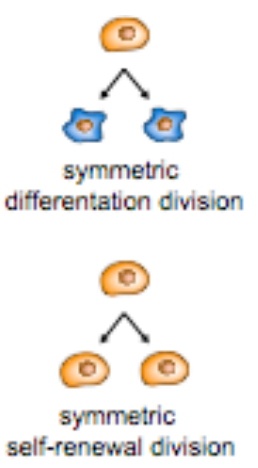
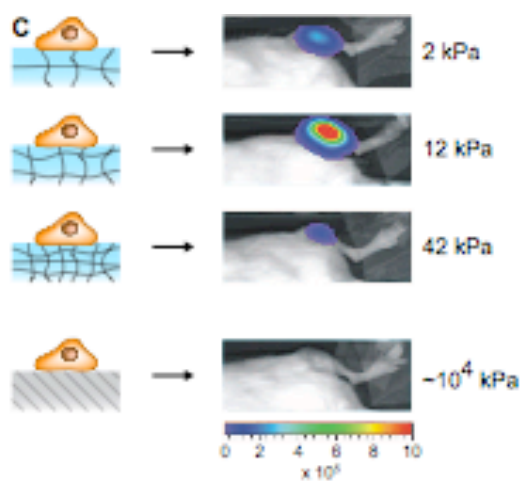
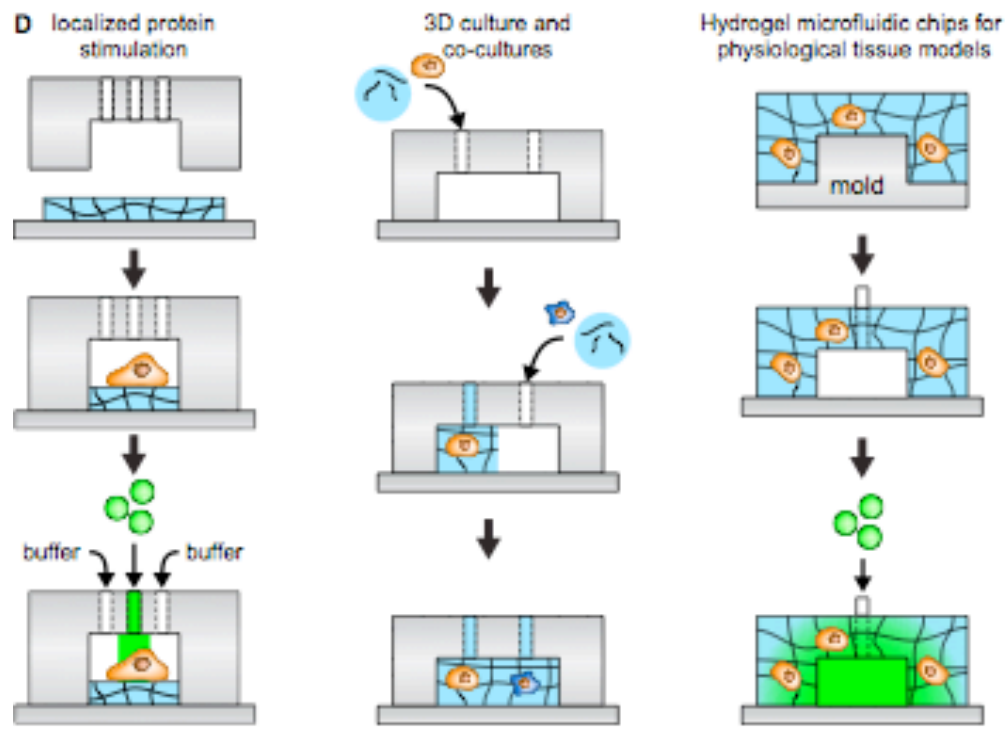

E High-throughput scroening of micro-environments

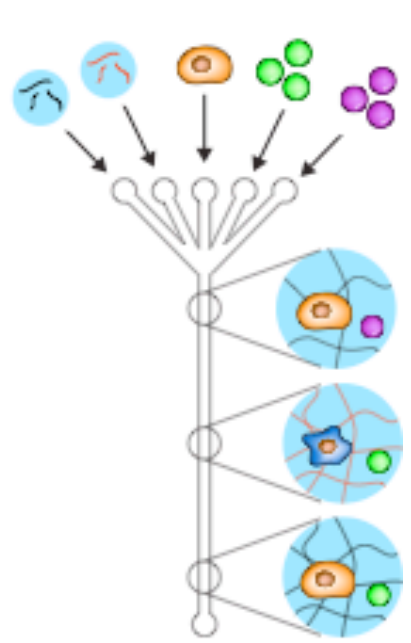

Figure 1: Application of biomaterials and microfluidics to mimic stem cell niches. (A) In vivo, stem cell are maintained in instructive microenvironments, or niches, which display short-ranged soluble signals, cell-cell interactions and tissue-specific ECMs. (B) These signals regulate stem cell behavior and in particular the delicate balance between self-renewal and differentiation. (C) In vitro systems mimicking stem cell niche properties (here: stiffness) can maintain stem cell function to some extend outside of a tissue. Adapted with permission from Reference [4*0]. (C) 2010 AAAS. (D) The integration of advanced biomaterials such as biomimetic hydrogels into microfluidics can augment microfluidic technologies by i) providing more physiological substrates for stem cell culture, ii) facilitating the precise fabrication of cocultures and iii) artificial tissue models. (E) Because microenvironments can be tightly controlled and rapidly modulated in microfluidic chips, miniaturization could render biomaterials amenable to the combinatorial screening of functional 3D artificial niches. 
Figure 2:
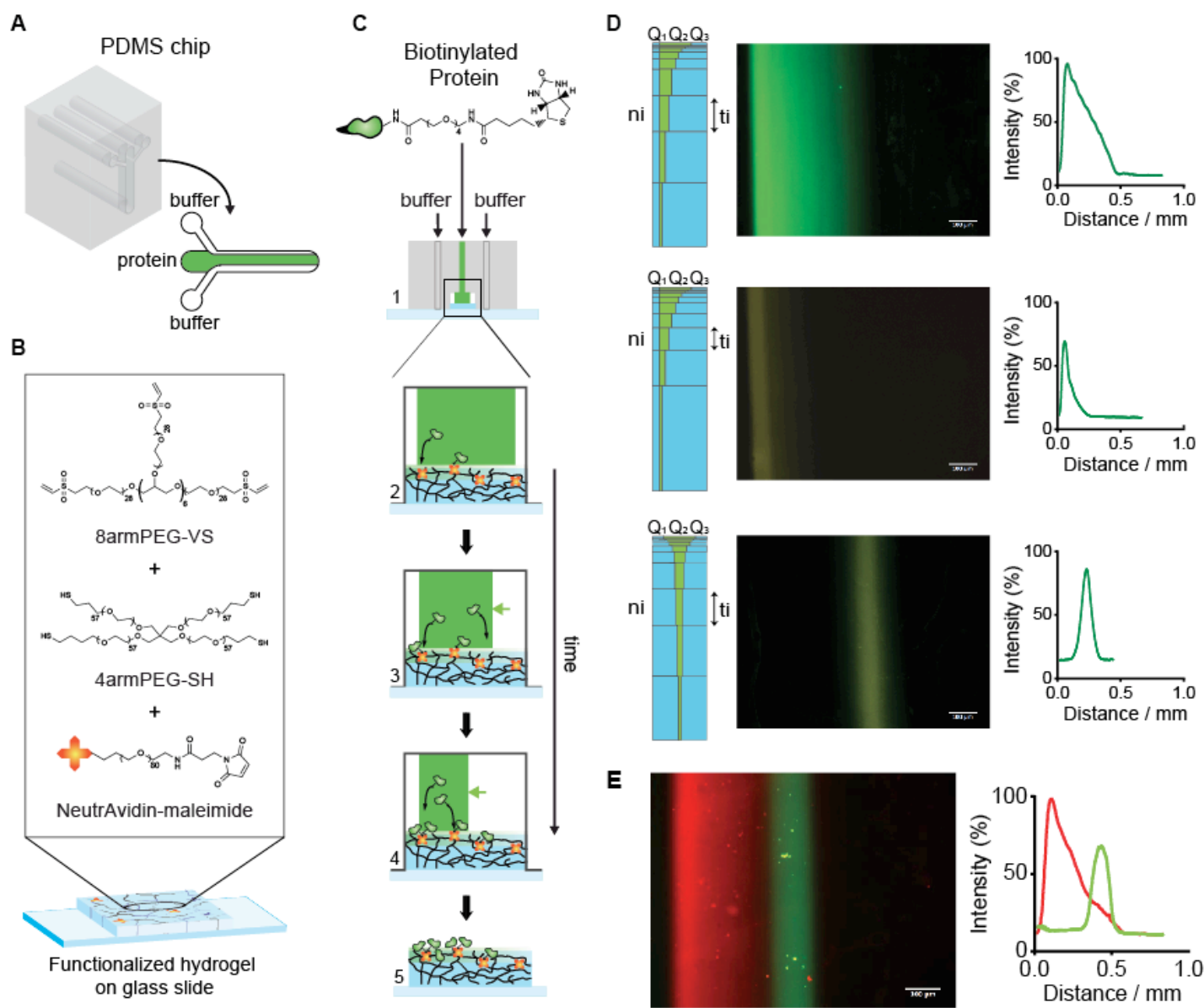

Figure 2: Schematic representation of the microfluidic patterning of functionalized PEG hydrogels. (A) Microfluidic chip for hydrodynamic flow focusing. (B) Formation of NeutrAvidin-conjugated hydrogels from aqueous precursor solutions containing multi-arm PEG macromers and 'PEGylated' NeutrAvidin. (C) Patterning of gels using hydrodynamic flow focusing. Step 1: the PDMS chip is pressed onto a glass slide bearing a thin hydrogel coating. Steps 2-4: gradient patterning by flow focusing. Step 5: the PDMS chip is removed for cell culture experiments. (D) Generation of three types of model gradients by hydrodynamic flow focusing of FITC-BSA-Biotin on NeutrAdvidinconjugated PEG gels. Fluorescent micrographs and graphical representations obtained by image analysis of a linear (top), exponential (middle) and Gaussian (bottom) gradients (scale bar $=100 \mu \mathrm{m}$ ). Schemes on the left of each gradient depict the step-wise flow focusing process with the two buffer streams $\left(Q_{1}\right.$ and $\left.Q_{3}\right)$ flanking the protein stream $Q_{2}$. Flow rates were maintained constant for a time $t_{i}$ for each step $n_{i}$. (E) Generation of gelimmobilized overlapping gradients by sequential hydrodynamic flow focusing. Fluorescence micrograph and measured fluorescence intensities showing a linear gradient of tethered DsRed-BSA-Biotin combined with a Gaussian gradient of FITC-labelled IgG (scale bar $=100 \mu \mathrm{m}$ ). Adapted with permission from Reference [21•]. (C) The Royal Society of Chemistry 2011 
Figure 3:

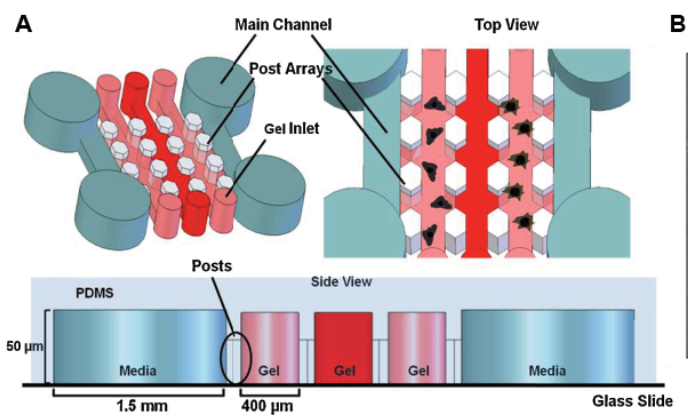

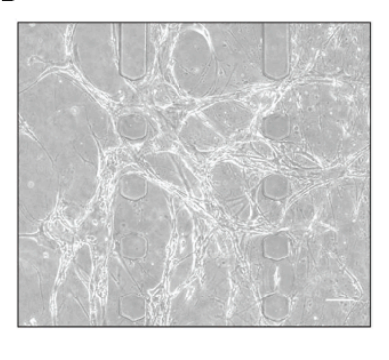

C

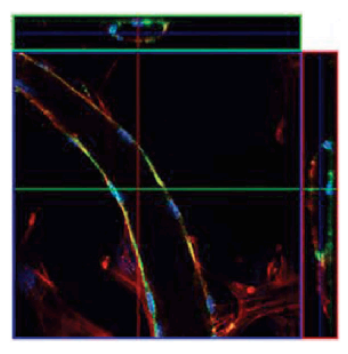

Figure 3: Microfluidics can also be explored to fabricate multi-layered hydrogels by physically confining the flow of hydrogel precursor solutions. (A, B) These 3D constructs permit precise co-cultures and can for example serve as models for tissue vascularization. (C) High magnification (63x) confocal images from day 14 co-cultures stained for F-actin and CD31. Scale bar $=50$ $\mu \mathrm{m}$. Reprinted with permission from [30•]. (c) 2010 John Wiley \& Sons

1. Morrison SJ, Spradling AC: Stem cells and niches: mechanisms that promote stem cell maintenance throughout life. Cell 2008, 132:598-611.

2. Moore KA, Lemischka IR: Stem cells and their niches. Science 2006, 311:1880-1885.

3. Engler AJ, Sen S, Sweeney HL, Discher DE: Matrix elasticity directs stem cell lineage specification. Cell 2006, 126:677-689.

•. 4. Gilbert PM, Havenstrite KL, Magnusson KEG, Sacco A, Leonardi NA, Kraft P, Nguyen NK, Thrun S, Lutolf MP, Blau HM: Substrate elasticity regulates skeletal muscle stem cell self-renewal in culture. Science 2010, 329:1078-1081.

5. Lutolf MP, Hubbell JA: Synthetic biomaterials as instructive extracellular microenvironments for morphogenesis in tissue engineering. Nat Biotechnol 2005, 23:47-55.

6. Discher DE, Mooney DJ, Zandstra PW: Growth factors, matrices, and forces combine and control stem cells. Science 2009, 324:1673-1677.

7. Lutolf MP, Gilbert PM, Blau HM: Designing materials to direct stem-cell fate. Nature 2009, 462:433-441.

8. Lee ST, Yun JI, Jo YS, Mochizuki M, van der Vlies AJ, Kontos S, Ihm JE, Lim $\mathrm{JM}$, Hubbell JA: Engineering integrin signaling for promoting embryonic stem cell self-renewal in a precisely defined niche. Biomaterials 2010, 31:1219-1226.

- 9. Kloxin AM, Kasko AM, Salinas CN, Anseth KS: Photodegradable hydrogels for dynamic tuning of physical and chemical properties. Science 2009, 324:59-63.

10. Lutolf MP: Integration column: artificial ECM: expanding the cell biology toolbox in 3D. Integr. Biol. 2009, 1:235-241.

11. Squires T, Quake S: Microfluidics: Fluid physics at the nanoliter scale. Rev Mod Phys 2005, 77:977-1026.

12. Kobel S, Lutolf MP: High-throughput methods to define complex stem cell niches. BioTechniques 2010, 48:IX-XXII.

13. Domachuk P, Tsioris K, Omenetto FG, Kaplan DL: Bio-microfluidics: biomaterials and biomimetic designs. Adv. Mater. 2010, 22:249-260. 
14. Takayama S, Ostuni E, LeDuc P, Naruse K, Ingber DE, Whitesides GM: Subcellular positioning of small molecules. Nature 2001, 411:1016.

15. Fung W-T, Beyzavi A, Abgrall P, Nguyen N-T, Li H-Y: Microfluidic platform for controlling the differentiation of embryoid bodies. Lab on a chip 2009, 9:2591-2595.

16. Lucchetta EM, Lee JH, Fu LA, Patel NH, Ismagilov RF: Dynamics of Drosophila embryonic patterning network perturbed in space and time using microfluidics. Nature 2005, 434:1134-1138.

17. Kim S, Kim HJ, Jeon NL: Biological applications of microfluidic gradient devices. Integr. Biol. 2010, 2:584-603.

18. Chung BG, Flanagan LA, Rhee SW, Schwartz PH, Lee AP, Monuki ES, Jeon $\mathrm{NL}$ : Human neural stem cell growth and differentiation in a gradientgenerating microfluidic device. Lab on a chip 2005, 5:401-406.

- 19. Cheung YK, Azeloglu EU, Shiovitz DA, Costa KD, Seliktar D, Sia SK: Microscale control of stiffness in a cell-adhesive substrate using microfluidics-based lithography. Angew Chem Int Ed Engl 2009, 48:71887192.

20. Cosson S, Kobel SA, Lutolf MP: Capturing Complex Protein Gradients on Biomimetic Hydrogels for Cell-Based Assays. Adv. Funct. Mater. 2009, 19:3411-3419.

-21. Allazetta S, Cosson S, Lutolf MP: Programmable microfluidic patterning of protein gradients on hydrogels. Chem Commun 2011, 47:191-193.

22. Selimović Š, Sim WY, Kim SB, Jang YH, Lee WG, Khabiry M, Bae H, Jambovane S, Hong JW, Khademhosseini A: Generating nonlinear concentration gradients in microfluidic devices for cell studies. Anal Chem 2011, 83:2020-2028.

-23. Chiu DT, Jeon NL, Huang S, Kane RS, Wargo CJ, Choi IS, Ingber DE, Whitesides GM: Patterned deposition of cells and proteins onto surfaces by using three-dimensional microfluidic systems. Proc Natl Acad Sci USA 2000, 97:2408-2413.

24. Liu J, Gao D, Li H-F, Lin J-M: Controlled photopolymerization of hydrogel microstructures inside microchannels for bioassays. Lab on a chip 2009, 9:1301-1305.

25. Cheung YK, Gillette BM, Zhong M, Ramcharan S, Sia SK: Direct patterning of composite biocompatible microstructures using microfluidics. Lab on a chip 2007, 7:574-579.

-26. Kunze A, Giugliano M, Valero A, Renaud P: Micropatterning neural cell cultures in 3D with a multi-layered scaffold. Biomaterials 2011, 32:20882098.

27. Wong AP, Perez-Castillejos R, Christopher Love J, Whitesides GM: Partitioning microfluidic channels with hydrogel to construct tunable 3D cellular microenvironments. Biomaterials 2008, 29:1853-1861.

28. Chung S, Sudo R, Mack PJ, Wan C-R, Vickerman V, Kamm RD: Cell migration into scaffolds under co-culture conditions in a microfluidic platform. Lab on a chip 2009, 9:269-275.

29. Huang CP, Lu J, Seon H, Lee AP, Flanagan LA, Kim H-Y, Putnam AJ, Jeon NL: Engineering microscale cellular niches for three-dimensional multicellular co-cultures. Lab on a chip 2009, 9:1740-1748.

- 30. Carrion B, Huang CP, Ghajar CM, Kachgal S, Kniazeva E, Jeon NL, Putnam AJ: Recreating the perivascular niche ex vivo using a microfluidic approach. Biotechnol Bioeng 2010, 107:1020-1028.

31. Blow N: Cell migration: our protruding knowledge. Nat Methods 2007, 4:589-594.

32. Kothapalli CR, Van Veen E, De Valence S, Chung S, Zervantonakis IK, Gertler FB, Kamm RD: A high-throughput microfluidic assay to study 
neurite response to growth factor gradients. Lab on a chip 2011, 11:497507.

33. Vickerman V, Blundo J, Chung S, Kamm R: Design, fabrication and implementation of a novel multi-parameter control microfluidic platform for three-dimensional cell culture and real-time imaging. Lab on a chip 2008, 8:1468-1477.

34. Choi NW, Cabodi M, Held B, Gleghorn JP, Bonassar LJ, Stroock AD: Microfluidic scaffolds for tissue engineering. Nature materials 2007, 6:908-915.

35. Cuchiara MP, Allen ACB, Chen TM, Miller JS, West JL: Multilayer microfluidic PEGDA hydrogels. Biomaterials 2010, 31:5491-5497.

36. Golden AP, Tien J: Fabrication of microfluidic hydrogels using molded gelatin as a sacrificial element. Lab on a chip 2007, 7:720-725.

37. Gillette BM, Jensen JA, Tang B, Yang GJ, Bazargan-Lari A, Zhong M, Sia SK: In situ collagen assembly for integrating microfabricated threedimensional cell-seeded matrices. Nature materials 2008, 7:636-640.

38. Haessler U, Kalinin Y, Swartz MA, Wu M: An agarose-based microfluidic platform with a gradient buffer for 3D chemotaxis studies. Biomedical microdevices 2009, 11:827-835.

39. Boland $T, X u T$, Damon $B$, Cui $X$ : Application of inkjet printing to tissue engineering. Biotechnol. J. 2006, 1:910-917.

40. Fedorovich NE, Alblas J, de Wijn JR, Hennink WE, Verbout AJ, Dhert WJA: Hydrogels as extracellular matrices for skeletal tissue engineering: state-of-the-art and novel application in organ printing. Tissue Eng 2007, 13:1905-1925.

41. Maerkl SJ: Integration column: Microfluidic high-throughput screening. Integr. Biol. 2009, 1:19-29.

42. Lii J, Hsu W-J, Parsa H, Das A, Rouse R, Sia SK: Real-time microfluidic system for studying mammalian cells in 3D microenvironments. Anal Chem 2008, 80:3640-3647.

43. Pregibon DC, Toner M, Doyle PS: Multifunctional encoded particles for high-throughput biomolecule analysis. Science 2007, 315:1393-1396.

44. Hwang DK, Oakey J, Toner M, Arthur JA, Anseth KS, Lee S, Zeiger A, Van Vliet KJ, Doyle PS: Stop-flow lithography for the production of shapeevolving degradable microgel particles. J Am Chem Soc 2009, 131:44994504.

45. Zeng S, Liu X, Xie H, Lin B: Basic Technologies for Droplet Microfluidics. Topics in current chemistry 2011.

• 46. Brouzes E, Medkova M, Savenelli N, Marran D, Twardowski M, Hutchison JB, Rothberg JM, Link DR, Perrimon N, Samuels ML: Droplet microfluidic technology for single-cell high-throughput screening. Proc Natl Acad Sci USA 2009, 106:14195-14200.

47. Chen AA, Underhill GH, Bhatia SN: Multiplexed, high-throughput analysis of 3D microtissue suspensions. Integr. Biol. 2010, 2:517-527.

- 48. Kumachev A, Greener J, Tumarkin E, Eiser E, Zandstra PW, Kumacheva E: High-throughput generation of hydrogel microbeads with varying elasticity for cell encapsulation. Biomaterials 2011, 32:1477-1483.

49. Trivedi V, Ereifej ES, Doshi A, Sehgal P, Vandevord PJ, Basu AS: Microfluidic encapsulation of cells in alginate capsules for high throughput screening. Conf Proc IEEE Eng Med Biol Soc 2009, 2009:70377040.

50. Choi C-H, Jung J-H, Rhee YW, Kim D-P, Shim S-E, Lee C-S: Generation of monodisperse alginate microbeads and in situ encapsulation of cell in microfluidic device. Biomedical microdevices 2007, 9:855-862. 
51. Chen W, Yang Y, Rinadi C, Zhou D, Shen AQ: Formation of supramolecular hydrogel microspheres via microfluidics. Lab on a chip 2009, 9:2947-2951.

• 52. Wang Y, Lin W-Y, Liu K, Lin RJ, Selke M, Kolb HC, Zhang N, Zhao X-Z, Phelps ME, Shen CKF, et al.: An integrated microfluidic device for largescale in situ click chemistry screening. Lab on a chip 2009, 9:2281-2285.

53. Shibata H, Heo YJ, Okitsu T, Matsunaga Y, Kawanishi T, Takeuchi S: Injectable hydrogel microbeads for fluorescence-based in vivo continuous glucose monitoring. Proc Natl Acad Sci USA 2010, 107:1789417898.

54. Bruzewicz DA, McGuigan AP, Whitesides GM: Fabrication of a modular tissue construct in a microfluidic chip. Lab on a chip 2008, 8:663-671.

55. Fok EYL, Zandstra PW: Shear-controlled single-step mouse embryonic stem cell expansion and embryoid body-based differentiation. Stem Cells 2005, 23:1333-1342.

• 56. Huh D, Matthews BD, Mammoto A, Montoya-Zavala M, Hsin HY, Ingber DE: Reconstituting organ-level lung functions on a chip. Science 2010, 328:1662-1668.

57. Qi H, Du Y, Wang L, Kaji H, Bae H, Khademhosseini A: Patterned differentiation of individual embryoid bodies in spatially organized 3D hybrid microgels. Adv. Mater. 2010, 22:5276-5281.

58. Pautot S, Wyart C, Isacoff EY: Colloid-guided assembly of oriented 3D neuronal networks. Nat Methods 2008, 5:735-740.

59. Sudo R, Chung S, Zervantonakis IK, Vickerman V, Toshimitsu Y, Griffith LG, Kamm RD: Transport-mediated angiogenesis in 3D epithelial coculture. FASEB J 2009, 23:2155-2164.

60. Méndez-Ferrer S, Michurina TV, Ferraro F, Mazloom AR, Macarthur BD, Lira SA, Scadden DT, Ma'ayan A, Enikolopov GN, Frenette PS: Mesenchymal and haematopoietic stem cells form a unique bone marrow niche. Nature 2010, 466:829-834.

1. Morrison SJ, Spradling AC: Stem cells and niches: mechanisms that promote stem cell maintenance throughout life. Cell 2008, 132:598-611.

2. Moore KA, Lemischka IR: Stem cells and their niches. Science 2006, 311:1880-1885.

3. Engler AJ, Sen S, Sweeney HL, Discher DE: Matrix elasticity directs stem cell lineage specification. Cell 2006, 126:677-689.

•- 4. Gilbert PM, Havenstrite KL, Magnusson KEG, Sacco A, Leonardi NA, Kraft P, Nguyen NK, Thrun S, Lutolf MP, Blau HM: Substrate elasticity regulates skeletal muscle stem cell self-renewal in culture. Science 2010, 329:1078-1081.

5. Lutolf MP, Hubbell JA: Synthetic biomaterials as instructive extracellular microenvironments for morphogenesis in tissue engineering. Nat Biotechnol 2005, 23:47-55.

6. Discher DE, Mooney DJ, Zandstra PW: Growth factors, matrices, and forces combine and control stem cells. Science 2009, 324:1673-1677.

7. Lutolf MP, Gilbert PM, Blau HM: Designing materials to direct stem-cell fate. Nature 2009, 462:433-441.

8. Lee ST, Yun JI, Jo YS, Mochizuki M, van der Vlies AJ, Kontos S, Ihm JE, Lim JM, Hubbell JA: Engineering integrin signaling for promoting embryonic stem cell self-renewal in a precisely defined niche. Biomaterials 2010, 31:1219-1226. 
- 9. Kloxin AM, Kasko AM, Salinas CN, Anseth KS: Photodegradable hydrogels for dynamic tuning of physical and chemical properties. Science 2009, 324:59-63.

10. Lutolf MP, Doyonnas R, Havenstrite K, Koleckar K, Blau HM: Perturbation of single hematopoietic stem cell fates in artificial niches. Integr. Biol. 2009, 1:59-69.

11. Squires T, Quake S: Microfluidics: Fluid physics at the nanoliter scale. Rev Mod Phys 2005, 77:977-1026.

12. Kobel S, Lutolf MP: High-throughput methods to define complex stem cell niches. BioTechniques 2010, 48:IX-XXII.

13. Domachuk P, Tsioris K, Omenetto FG, Kaplan DL: Bio-microfluidics: biomaterials and biomimetic designs. Adv. Mater. 2010, 22:249-260.

14. Takayama S, Ostuni E, LeDuc P, Naruse K, Ingber DE, Whitesides GM: Subcellular positioning of small molecules. Nature 2001, 411:1016.

15. Fung W-T, Beyzavi A, Abgrall P, Nguyen N-T, Li H-Y: Microfluidic platform for controlling the differentiation of embryoid bodies. Lab on a chip 2009 , 9:2591-2595.

16. Lucchetta EM, Lee JH, Fu LA, Patel NH, Ismagilov RF: Dynamics of Drosophila embryonic patterning network perturbed in space and time using microfluidics. Nature 2005, 434:1134-1138.

17. Kim S, Kim HJ, Jeon NL: Biological applications of microfluidic gradient devices. Integr. Biol. 2010, 2:584-603.

18. Chung BG, Flanagan LA, Rhee SW, Schwartz PH, Lee AP, Monuki ES, Jeon NL: Human neural stem cell growth and differentiation in a gradientgenerating microfluidic device. Lab on a chip 2005, 5:401-406.

- 19. Cheung YK, Azeloglu EU, Shiovitz DA, Costa KD, Seliktar D, Sia SK: Microscale control of stiffness in a cell-adhesive substrate using microfluidics-based lithography. Angew Chem Int Ed Engl 2009, 48:71887192.

20. Cosson S, Kobel SA, Lutolf MP: Capturing Complex Protein Gradients on Biomimetic Hydrogels for Cell-Based Assays. Adv. Funct. Mater. 2009, 19:3411-3419.

-21. Allazetta S, Cosson S, Lutolf MP: Programmable microfluidic patterning of protein gradients on hydrogels. Chem Commun 2011, 47:191-193.

22. Selimović Š, Sim WY, Kim SB, Jang YH, Lee WG, Khabiry M, Bae H, Jambovane S, Hong JW, Khademhosseini A: Generating nonlinear concentration gradients in microfluidic devices for cell studies. Anal Chem 2011, 83:2020-2028.

- 23. Chiu DT, Jeon NL, Huang S, Kane RS, Wargo CJ, Choi IS, Ingber DE, Whitesides GM: Patterned deposition of cells and proteins onto surfaces by using three-dimensional microfluidic systems. Proc Natl Acad Sci USA 2000, 97:2408-2413.

24. Liu J, Gao D, Li H-F, Lin J-M: Controlled photopolymerization of hydrogel microstructures inside microchannels for bioassays. Lab on a chip 2009, 9:1301-1305.

25. Cheung YK, Gillette BM, Zhong M, Ramcharan S, Sia SK: Direct patterning of composite biocompatible microstructures using microfluidics. Lab on a chip 2007, 7:574-579.

-26. Kunze A, Giugliano M, Valero A, Renaud P: Micropatterning neural cell cultures in 3D with a multi-layered scaffold. Biomaterials 2011, 32:20882098.

27. Wong AP, Perez-Castillejos R, Christopher Love J, Whitesides GM: Partitioning microfluidic channels with hydrogel to construct tunable 3D cellular microenvironments. Biomaterials 2008, 29:1853-1861. 
28. Chung S, Sudo R, Mack PJ, Wan C-R, Vickerman V, Kamm RD: Cell migration into scaffolds under co-culture conditions in a microfluidic platform. Lab on a chip 2009, 9:269-275.

29. Huang CP, Lu J, Seon H, Lee AP, Flanagan LA, Kim H-Y, Putnam AJ, Jeon NL: Engineering microscale cellular niches for three-dimensional multicellular co-cultures. Lab on a chip 2009, 9:1740-1748.

- 30. Carrion B, Huang CP, Ghajar CM, Kachgal S, Kniazeva E, Jeon NL, Putnam $\mathrm{AJ}$ : Recreating the perivascular niche ex vivo using a microfluidic approach. Biotechnol Bioeng 2010, 107:1020-1028.

31. Blow N: Cell migration: our protruding knowledge. Nat Methods 2007, 4:589-594.

32. Kothapalli CR, Van Veen E, De Valence S, Chung S, Zervantonakis IK, Gertler FB, Kamm RD: A high-throughput microfluidic assay to study neurite response to growth factor gradients. Lab on a chip 2011, 11:497507.

33. Vickerman V, Blundo J, Chung S, Kamm R: Design, fabrication and implementation of a novel multi-parameter control microfluidic platform for three-dimensional cell culture and real-time imaging. Lab on a chip 2008, 8:1468-1477.

34. Choi NW, Cabodi M, Held B, Gleghorn JP, Bonassar LJ, Stroock AD: Microfluidic scaffolds for tissue engineering. Nature materials 2007, 6:908-915.

35. Cuchiara MP, Allen ACB, Chen TM, Miller JS, West JL: Multilayer microfluidic PEGDA hydrogels. Biomaterials 2010, 31:5491-5497.

36. Golden AP, Tien J: Fabrication of microfluidic hydrogels using molded gelatin as a sacrificial element. Lab on a chip 2007, 7:720-725.

37. Gillette BM, Jensen JA, Tang B, Yang GJ, Bazargan-Lari A, Zhong M, Sia SK: In situ collagen assembly for integrating microfabricated threedimensional cell-seeded matrices. Nature materials 2008, 7:636-640.

38. Haessler U, Kalinin Y, Swartz MA, Wu M: An agarose-based microfluidic platform with a gradient buffer for 3D chemotaxis studies. Biomedical microdevices 2009, 11:827-835.

39. Boland $T, X u T$, Damon $B$, Cui $X$ : Application of inkjet printing to tissue engineering. Biotechnol. J. 2006, 1:910-917.

40. Fedorovich NE, Alblas J, de Wijn JR, Hennink WE, Verbout AJ, Dhert WJA: Hydrogels as extracellular matrices for skeletal tissue engineering: state-of-the-art and novel application in organ printing. Tissue Eng 2007, 13:1905-1925.

41. Maerkl SJ: Integration column: Microfluidic high-throughput screening. Integr. Biol. 2009, 1:19-29.

42. Lii J, Hsu W-J, Parsa H, Das A, Rouse R, Sia SK: Real-time microfluidic system for studying mammalian cells in 3D microenvironments. Anal Chem 2008, 80:3640-3647.

43. Pregibon DC, Toner M, Doyle PS: Multifunctional encoded particles for high-throughput biomolecule analysis. Science 2007, 315:1393-1396.

44. Hwang DK, Oakey J, Toner M, Arthur JA, Anseth KS, Lee S, Zeiger A, Van Vliet KJ, Doyle PS: Stop-flow lithography for the production of shapeevolving degradable microgel particles. J Am Chem Soc 2009, 131:44994504.

45. Zeng S, Liu X, Xie H, Lin B: Basic Technologies for Droplet Microfluidics. Topics in current chemistry 2011.

• 46. Brouzes E, Medkova M, Savenelli N, Marran D, Twardowski M, Hutchison JB, Rothberg JM, Link DR, Perrimon N, Samuels ML: Droplet microfluidic technology for single-cell high-throughput screening. Proc Natl Acad Sci USA 2009, 106:14195-14200. 
47. Chen AA, Underhill GH, Bhatia SN: Multiplexed, high-throughput analysis of 3D microtissue suspensions. Integr. Biol. 2010, 2:517-527.

- 48. Kumachev A, Greener J, Tumarkin E, Eiser E, Zandstra PW, Kumacheva E: High-throughput generation of hydrogel microbeads with varying elasticity for cell encapsulation. Biomaterials 2011, 32:1477-1483.

49. Trivedi V, Ereifej ES, Doshi A, Sehgal P, Vandevord PJ, Basu AS: Microfluidic encapsulation of cells in alginate capsules for high throughput screening. Conf Proc IEEE Eng Med Biol Soc 2009, 2009:70377040.

50. Choi C-H, Jung J-H, Rhee YW, Kim D-P, Shim S-E, Lee C-S: Generation of monodisperse alginate microbeads and in situ encapsulation of cell in microfluidic device. Biomedical microdevices 2007, 9:855-862.

51. Chen W, Yang Y, Rinadi C, Zhou D, Shen AQ: Formation of supramolecular hydrogel microspheres via microfluidics. Lab on a chip 2009, 9:2947-2951.

• 52. Wang Y, Lin W-Y, Liu K, Lin RJ, Selke M, Kolb HC, Zhang N, Zhao X-Z, Phelps ME, Shen CKF, et al:: An integrated microfluidic device for largescale in situ click chemistry screening. Lab on a chip 2009, 9:2281-2285.

53. Shibata H, Heo YJ, Okitsu T, Matsunaga Y, Kawanishi T, Takeuchi S: Injectable hydrogel microbeads for fluorescence-based in vivo continuous glucose monitoring. Proc Natl Acad Sci USA 2010, 107:1789417898.

54. Bruzewicz DA, McGuigan AP, Whitesides GM: Fabrication of a modular tissue construct in a microfluidic chip. Lab on a chip 2008, 8:663-671.

55. Fok EYL, Zandstra PW: Shear-controlled single-step mouse embryonic stem cell expansion and embryoid body-based differentiation. Stem Cells 2005, 23:1333-1342.

•• 56. Huh D, Matthews BD, Mammoto A, Montoya-Zavala M, Hsin HY, Ingber DE: Reconstituting organ-level lung functions on a chip. Science 2010, 328:1662-1668.

57. Qi H, Du Y, Wang L, Kaji H, Bae H, Khademhosseini A: Patterned differentiation of individual embryoid bodies in spatially organized 3D hybrid microgels. Adv. Mater. 2010, 22:5276-5281.

58. Pautot S, Wyart C, Isacoff EY: Colloid-guided assembly of oriented 3D neuronal networks. Nat Methods 2008, 5:735-740.

59. Sudo R, Chung S, Zervantonakis IK, Vickerman V, Toshimitsu Y, Griffith LG, Kamm RD: Transport-mediated angiogenesis in 3D epithelial coculture. FASEB J 2009, 23:2155-2164.

60. Méndez-Ferrer S, Michurina TV, Ferraro F, Mazloom AR, Macarthur BD, Lira SA, Scadden DT, Ma'ayan A, Enikolopov GN, Frenette PS: Mesenchymal and haematopoietic stem cells form a unique bone marrow niche. Nature 2010, 466:829-834. 